\title{
What makes it so difficult for nurses to coach patients in shared decision making? A process evaluation
}

Citation for published version (APA):

Lenzen, S. A., Daniels, R., van Bokhoven, M. A., van der Weijden, T., \& Beurskens, A. (2017). What makes it so difficult for nurses to coach patients in shared decision making? A process evaluation. International Journal of Nursing Studies, 80, 1-11. https://doi.org/10.1016/j.jinurstu.2017.12.005

Document status and date:

Published: 01/04/2017

DOI:

10.1016/j.jinurstu.2017.12.005

Document Version:

Publisher's PDF, also known as Version of record

Document license:

Taverne

Please check the document version of this publication:

- A submitted manuscript is the version of the article upon submission and before peer-review. There can be important differences between the submitted version and the official published version of record.

People interested in the research are advised to contact the author for the final version of the publication, or visit the DOI to the publisher's website.

- The final author version and the galley proof are versions of the publication after peer review.

- The final published version features the final layout of the paper including the volume, issue and page numbers.

Link to publication

\footnotetext{
General rights rights.

- You may freely distribute the URL identifying the publication in the public portal. please follow below link for the End User Agreement:

www.umlib.nl/taverne-license

Take down policy

If you believe that this document breaches copyright please contact us at:

repository@maastrichtuniversity.nl

providing details and we will investigate your claim.
}

Copyright and moral rights for the publications made accessible in the public portal are retained by the authors and/or other copyright owners and it is a condition of accessing publications that users recognise and abide by the legal requirements associated with these

- Users may download and print one copy of any publication from the public portal for the purpose of private study or research.

- You may not further distribute the material or use it for any profit-making activity or commercial gain

If the publication is distributed under the terms of Article $25 \mathrm{fa}$ of the Dutch Copyright Act, indicated by the "Taverne" license above, 


\title{
What makes it so difficult for nurses to coach patients in shared decision making? A process evaluation
}

\author{
Stephanie Anna Lenzen ${ }^{\mathrm{a}, \mathrm{b}, *}$, Ramon Daniëls ${ }^{\mathrm{a}}$, Marloes Amantia van Bokhoven ${ }^{\mathrm{b}}$, \\ Trudy van der Weijden ${ }^{\mathrm{b}}$, Anna Beurskens $\mathrm{s}^{\mathrm{a}, \mathrm{b}}$ \\ ${ }^{a}$ Research Centre for Autonomy and Participation for People with a Chronic Illness, Zuyd University of Applied Sciences, Heerlen, The Netherlands \\ ${ }^{\mathrm{b}}$ Department of Family Medicine, CAPHRI School for Public Health and Primary Care, Maastricht University, Maastricht, The Netherlands
}

\section{A R T I C L E I N F O}

\section{Keywords:}

Action-planning

Goal setting

Health coaching

Practice nurses

Self-management

Shared decision making

\begin{abstract}
A B S T R A C T
Background: Primary care nurses play a crucial role in coaching patients in shared decision making about goals and actions. This presents a challenge to practice nurses, who are frequently used to protocol-based working routines. Therefore, an approach was developed to support nurses to coach patients in shared decision making. Objectives: To investigate how the approach was implemented and experienced by practice nurses and patients. Design: A process evaluation was conducted using quantitative and qualitative methods.

Settings/Participants: Fifteen female practice nurses (aged between 28 and 55 years), working with people suffering from diabetes, COPD, asthma and/or cardiovascular diseases, participated. Nurses were asked to apply the approach to their chronically ill patients and to recruit patients $(n=10)$ willing to participate in an interview or an audio-recording of a consultation $(n=13)$; patients ( 13 women, 10 men) were aged between 41 and 88 years and suffered from diabetes, COPD or cardiovascular diseases.

Methods: The approach involved a framework for shared decision making about goals and actions, a tool to explore the patient perspective, a patient profiles model and a training course. Interviews ( $\mathrm{n}=15$ ) with nurses, a focus group with nurses $(n=9)$ and interviews with patients $(n=10)$ were conducted. Nurses filled in a questionnaire about their work routine before, during and after the training course. They were asked to deliver audiotapes of their consultations $(\mathrm{n}=13)$.

Results: Overall, nurses felt that the approach supported them to coach patients in shared decision making. Nurses had become more aware of their own attitudes and learning needs and reported to have had more indepth discussions with patients. The on-the-job coaching was experienced as valuable. However, nurses struggled to integrate the approach in routine care. They experienced the approach as different to their protocol-based routines and expressed the importance of receiving support and the need for integration of the approach into the family physician practice.

Conclusion: This study shows that changing practice nurses' role from medical experts to coaches in shared decision making is very complex and requires paying attention to skills and attitudes, as well as to contextual factors. Our results indicate that more time and training might be needed for this role transition. Moreover, it might be worthwhile to focus on organizational learning, in order to increase an organization's capacity to change work routines in a collaborative process. Future research into the development and evaluation of health coaching approaches, focusing on shared decision making, is necessary.
\end{abstract}

\section{What is already known about the topic?}

- Practice nurses working in primary care family medicine play a crucial role in coaching patients with chronic conditions in shared decision making about goals and actions.

- Coaching patients presents a challenge to practice nurses as they are used to a more medical protocol-based working method.

- Practice nurses might need more training in shared decision making about goals and actions, especially focusing on exploring the patient's perspective from an holistic point of view, setting goals and actions explicitly, and tailoring decision making to the individual patient.

\footnotetext{
* Corresponding author. S.A. Lenzen (MSc) Research Centre for Autonomy and Participation for People with a Chronic Illness Zuyd University of Applied Sciences Nieuw Eyckholt 300, 6419 DJ Heerlen, The Netherlands.

E-mail address: stephanie.lenzen@zuyd.nl (S.A. Lenzen).
} 


\section{What this paper adds}

- This paper presents an approach and a corresponding training course aiming to support practice nurses in coaching patients in shared decision making (involving several tools to put shared decision making into practice, to explore the patient perspective and to tailor coaching).

- Although the approach under study was experienced as valuable by practice nurses and patients, practice nurses experienced the approach as very different to their protocol-based work routines and struggled to integrate it in routine care. Practice nurses experienced a need for more support and integration of the approach into the family physician practice.

- Changing practice nurses' role from medical experts to health coaches might requires attention to nurses' skills and attitudes, as well as to organizational learning, aiming to involve the whole family medicine practice to learn and change work routines in a collaborative process.

\section{Background}

The prevalence of chronic conditions such as diabetes and chronic pulmonary disease is still increasing worldwide (WHO, 2010; Yach et al., 2004). Improvements in medical care and technological advances have meant that patients with chronic diseases have a longer life expectancy and use primary care long-term (Fortin et al., 2004). Selfmanagement, defined as 'the degree to which a patient with a chronic condition is able to and wants to control his own daily life', is an essential part of primary chronic care (Lorig and Holman, 2003; Wagner et al., 2005; Barr et al., 2003). Self-management involves not only medical management, but also maintaining and changing life roles (social self-management) and dealing with emotional consequences of the disease (emotional self-management) (Corbin and Strauss, 1988).

Core elements of self-management are goal setting and action planning. Goal setting is defined as 'a process in which health care professionals and patients agree on a health-related goal'. Action planning is defined as 'agreeing on a course of actions to reach the goal, including questions like what, when, where and how often' (Bodenheimer and Handley, 2009; Lorig, 2006). In medical settings, shared decision making is widely recognized as a way to support patients in making health care choices (Elwyn et al., 2012; Stiggelbout et al., 2012). It is mostly applied in curative settings and in situations with a limited number of preference-sensitive treatment options (van de Pol et al., 2016). Currently, there is increased interest in applying shared decision making in chronic care. As the nature of chronic care is dynamic and progressive, shared decision making in chronic care is characterized by a continuous counselling dialog between health professional and patient (van de Pol et al., 2016; De Sutter et al., 2013; Lenzen et al., 2015; Friesen-Storms et al., 2015). Moreover, as chronic conditions impact on patients' everyday lives, exploration of patients' situations and their goals is a precondition to making shared decisions on treatment and lifestyle (van de Pol et al., 2016; De Sutter et al., 2013; Lenzen et al., 2015; Friesen-Storms et al., 2015).

To become a collaborative partner in decision making, patients might need support from their health professionals. The literature frequently uses the term health coaching (Bennett et al., 2010; Ghorob, 2013; Willard-Grace et al., 2015). Health coaching is defined as 'helping patients gain knowledge, skills, tools and confidence to become active participants in their care' (Bennett et al., 2010). A health coach seeks to enhance patient motivation by supporting the patients in setting goals that fit their situation and motivation (Willard-Grace et al., 2015). Health coaching is always tailored to what is important to the patient, to what the patient wants to learn and to his/her motivation (Ghorob, 2013). It has the potential to improve chronic care and assist professionals struggling with insufficient time (Bennett et al., 2010; Willard-Grace et al., 2015).
In Dutch primary care, as in many other developed countries, practice nurses often play a crucial role in coaching patients with chronic diseases. They see patients with chronic diseases on a regular basis and are mandated by the family physicians to apply most of the long-term condition management (Van Dijk-de Vries, 2015; WillardGrace et al., 2015; Laurent et al., 2005). However, practice nurses in family medicine usually work according to fixed protocols that mainly focus on medical management. Their tasks often involve monitoring and recording disease-specific outcomes and they sometimes regard providing self-management support as time-consuming and increasing their workload (Heiligers et al., 2012; Kennedy et al., 2014; Lenzen et al., 2016). As this medical, protocol-based way of working conflicts with the fundamental principles of health coaching and shared decision making, practice nurses might need special training and support to be able to coach patients in shared decision making (Kennedy et al., 2014).

Using principles of Intervention Mapping, we developed an approach to support practice nurses in coaching their patients in shared decision making about goals and actions (Bartholomew et al., 2011). During the need assessment phase, involving several qualitative studies (including a focus group study and an observational study), a scoping review and action research, we found that nurses mostly struggled and lacked tools to explore the patient's perceptive from an holistic point of view, to explicitly set goals, and to tailor their working methods to individual patients (Lenzen et al., 2015, 2016, 2017).

Although literature is available about the importance and complexity of coaching patients in shared decision making, little information is to be found about the complexity for practice nurses to learn this skill in the primary care context (Bennett et al., 2010; Ghorob, 2013, Willard-Grace et al., 2015). We therefore investigated how our approach, focusing on supporting nurses to coach patients in shared decision making about goals and actions, works in practice. We did this by examining how the approach was implemented and which important factors contributed to its success. We conducted a process evaluation based on the Medical Research Council Process Evaluation Framework (Moore et al., 2015) and formulated the following research questions:

1. To what extent was the approach implemented?

2. How did professionals experience the approach (including the training course)?

3. How did professionals describe their learning process?

4. How did patients experience the approach?

\section{Methods}

We conducted a process evaluation using quantitative and qualitative methods.

\subsection{The approach}

We had developed the approach together with experts, professionals, patients and a regional family medicine organization. The approach is based on the following key principles:

1) Shared decision making is central to goal setting and action planning, asking for:

a. a holistic exploration of the patient's perspective;

b. tailored coaching of the patient.

2) Coaching patients in shared decision making about goals and actions is a complex skill for professionals and requires extensive training.

Therefore, our overall goal was to train practice nurses in coaching patients to actively engage in decision making about goals and actions. Three performance objectives with associated behavioral determinants were formulated (see Table 1).

The approach included a framework for shared decision making about goals and actions, a tool to explore the patient's perspective (the so-called 4-circles tool), a patient profiles model and a training course 
Table 1

Performance objectives and behavioral determinants.

\begin{tabular}{|c|c|}
\hline Performance objectives & Behavioral determinants \\
\hline $\begin{array}{l}\text { Practice nurses explore the patient's perspective from a } \\
\text { holistic point of view. }\end{array}$ & $\begin{array}{l}\text { Inviting the patient to ask questions or raise points for discussion. } \\
\text { Exploring the patient's experiences (of medical symptoms, impact of the condition on everyday life/work, } \\
\text { emotions, coping with the condition and support from the environment). }\end{array}$ \\
\hline $\begin{array}{l}\text { Practice nurses explicitly formulate goals and actions } \\
\text { together with the patient. }\end{array}$ & $\begin{array}{l}\text { Formulating goals and actions explicitly. } \\
\text { Deciding together on goals and actions. } \\
\text { Writing down goals and actions. }\end{array}$ \\
\hline Practice nurses tailor their coaching to the patient. & $\begin{array}{l}\text { Exploring patient profile (based on patients' perceived control and acceptance). } \\
\text { Tailoring goal setting and action planning to the patient's profile (e.g. number of goals and actions, difficulty of } \\
\text { goals and actions). }\end{array}$ \\
\hline
\end{tabular}

for nurses.

The framework for shared decision making about goals and actions intended to help professionals in going through the process of goal setting and action planning. It was developed in collaboration with the Dutch National Health Care Institute, the Dutch College of General Practitioners and a national primary care organization and includes tools that professionals can use throughout the process, and presents case examples. It is based on existing theories about shared decision making (Elwyn et al., 2012) and especially adds the phase of goal setting to the phase of making shared decisions about actions (see Fig. 1).

The 4-circles tool (see Fig. 2) aimed to support professionals in exploring patients' current and desired situation (with regard to health, everyday life, social/physical environment, and coping strategies), their motivation and readiness to change. It is meant to help patients gain insight into their own situation, making it possible to set goals accordingly. The tool is based on the framework of the International Classification of Functioning, Disability and Health (ICF) (WHO, 2010) as this framework presents a holistic approach to health, recognizing the interrelation between health and health-related domains.

In addition, the approach included a patient profiles model to support professionals in exploring patients' motivation and readiness to set goals and make action plans and to tailor their coaching accordingly (see Fig. 3). The model is based on the theory-based patient typology by Bloem \& Stalpers (Bloem and Stalpers, 2012). This typology focuses on the role of the subjective experience of health as a motivator of patients' health-related behaviors. In the model the patients' subjective experiences of health is determined by the perceived control over their health situation and by the acceptance of their health situation. Based on a combination of these two determinants, four profiles have been identified (Bloem and Stalpers, 2012). For practical use, these four profiles have been translated into personas by Dubois \& van Rij (Dubois and Van Rij, 2016). Typical behaviors for each persona have been described, as well as ways for practice nurses to adjust their coaching to the needs of each persona.

The training course focused on training professionals in coaching patients in shared decision making. Furthermore, the training aimed to stimulate practice nurses to recurrently reflect on their work routine and their work-related attitudes. The training consisted of a one-day training session (eight hours, week one), individual on-the job-coaching (four hours, week four) and a follow-up meeting (four hours, week eight). Moreover, participants were encouraged to contact the trainer for questions or further advice at any time. The trainer was a professional coach with 20 years of work experience in the field of coaching. During the one-day training session, participants were provided with an introduction to health coaching and shared decision making. By means of discussions, role-plays and demonstrations, participants were trained in exploring the patient's perspective, shared decision making about goals and actions and tailoring shared decision making to the patient's needs. Nurses learned techniques to self-reflect their behaviors, pitfalls and strengths and they were provided with a workbook. The on-the-job coaching took place three weeks after the one-day training session. The coach attended three consultations of each nurse, and had a coaching session (30-60 $\mathrm{min}$ ) with the nurse immediately after the consultations. During the consultations the coach observed the nurse and frequently used role-modeling to demonstrate skills. In the coaching sessions the nurse and the coach reflected on the consultations and set individual goals for the nurse. The follow-up meetings focused on the nurses' experiences and on coping with patients' resistance to shared decision making. The training course was organized by the regional family medicine organization, which plays a crucial role in defining the practice nurses' role in primary care and their education. Representatives of the family medicine organization were present at the one-day training session and the follow-up meeting, to support nurses in making decisions regarding the flexible use of the usual clinical protocols (i.e. tailoring shared decision making to the patient's context and individual change process, instead of sticking to fixed protocols).

\subsection{Procedure and inclusion criteria}

We included practice nurses who (a) worked with chronically ill patients in family medicine, and (b) had at least one year of work experience. They were recruited by the regional family medicine organization. The organization especially recruited nurses who had shown interest in training about shared decision making before, as we

\begin{tabular}{|c|c|}
\hline Elements & Explanation \\
\hline 1 Preparation & $\begin{array}{l}\text { Informing the patient about the aim of the consultation. } \\
\text { Inviting the patient to ask questions or raise points for discussion. }\end{array}$ \\
\hline $\begin{array}{l}2 \text { Goal setting } \\
\text { A Exploration } \\
\text { B Information giving } \\
\text { C Formulating goals }\end{array}$ & $\begin{array}{l}\text { Exploring the patient's current and desired situation. } \\
\text { Giving information tailored to the patient. } \\
\text { Supporting the patient in formulating feasible goals. }\end{array}$ \\
\hline $\begin{array}{l}3 \text { Action planning } \\
\text { A Choice talk } \\
\text { B Option talk } \\
\text { C Decision talk }\end{array}$ & $\begin{array}{l}\text { Making sure the patient knows that he/she has a choice. } \\
\text { Discussing possible options for actions with the patient. } \\
\text { Deciding on actions together with the patient. }\end{array}$ \\
\hline 4 Evaluation & $\begin{array}{l}\text { Continuously reflecting on the patient's progress, and adjusting } \\
\text { goals and actions. }\end{array}$ \\
\hline
\end{tabular}

Fig. 1. Framework for shared decision making about goals and actions. 


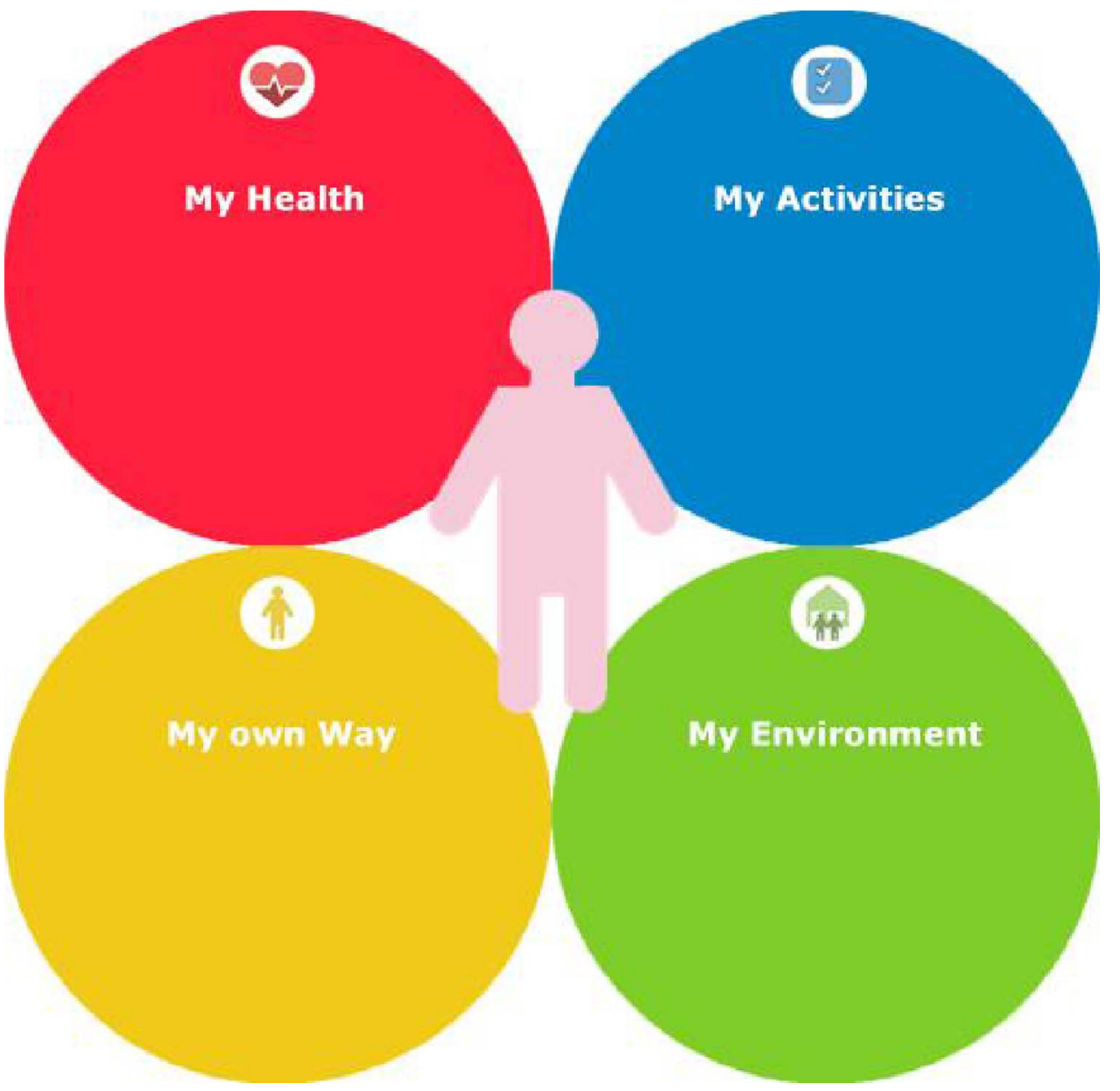

Fig. 2. The 4-cirlces tool.

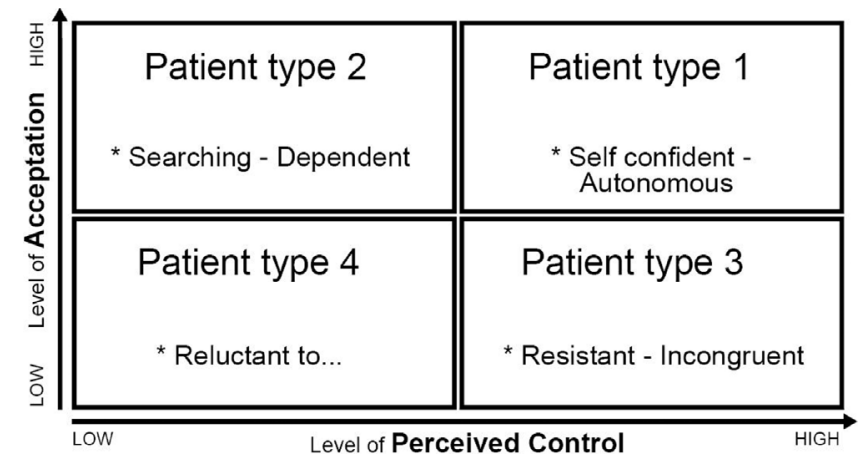

Fig. 3. Patient profiles model.

expected them to be more motivated to implement the approach into practice. Twenty practice nurses were invited to participate; 15 agreed to do so. Nine practice nurses were trained during September and October 2015 and six practice nurses were trained between May and June 2016. After the training, nurses were asked to apply the approach to all their chronically ill patients and to recruit patients willing to participate in the research. Patients were included if they had been seen by a practice nurse after the latter had attended the training course, and provided they were able to speak Dutch.

\subsection{Data collection}

We collected data from nurses and patients by means of qualitative and quantitative methods (see Table S1 (Supplementary material) for an overview of data collection methods for each research question, and
Table S2 (Supplementary material) for an overview of the timing of the data collection).

\subsubsection{Quantitative methods}

Quantitative measurements served to answer the first research question. We asked practice nurses to fill in a questionnaire about their current working method with regard to shared decision making about goals and actions. This was asked three times (before, during and after the training course ( $\mathrm{t} 0, \mathrm{t} 1$ and $\mathrm{t} 2$, respectively)). They were also asked to hand in 1 or 2 audiotapes of consultations in which they had applied the approach, without further selection criteria. The measurement instruments for the quantitative data collection had been developed by the research team based on the behavioral objectives formulated for the approach (see Table 1). The questionnaire for practice nurses included two parts (Part 1: current working method with regard to shared decision making about goals, and Part 2: current working method with regard to shared decision making about actions). For Part 2 we used the items of the Shared Decision Making Questionnaire-Physician version (SDM-Q-Doc) (Kriston et al., 2010). The SDM-Q-Doc measures professionals' perception of the extent of shared decision making during consultations, and has been found reliable (Rodenburg-Vandenbussche et al., 2015). We added one item to the SDM-Q-Doc, about documenting actions, as we regarded this as a critical behavioral factor for the approach within the needs assessment phase (Lenzen et al., 2016). Parts 1 and 2 had previously been pilot-tested for clarity with 3 practice nurses.

\subsubsection{Qualitative methods}

All practice nurses were invited to participate in individual interviews. Nurses who participated in the first training course were asked to take part in a focus group interview. In the individual interviews, and 
during the focus group interview, the nurses were questioned about their experience with the approach and the training course. During the individual interviews, the nurses were also encouraged to reflect on their learning process, on what they had learned, on what had hampered or supported them in their learning process, and what they still wanted to learn. In the interviews with patients, patients were asked about their experience of the most recent consultation with their practice nurses.

\subsection{Data analysis}

Quantitative data were analyzed using descriptive statistics (means, median) using Statistical Software package for Social Sciences (SPSS) version 23. To analyze the audio tapes we developed an observation form based on the first two main behavioral objectives (exploration of the patient's perspective and explicit formulation of goals and action plans) (see Table 1).

All qualitative data were recorded and transcribed verbatim. We applied content analysis and used Nvivo 10 as supportive software (Hsieh and Shannon, 2005; Mortelmans, 2007). We started with open coding, followed by axial coding, in which codes were grouped into categories (Hsieh and Shannon, 2005). Several strategies were used to enhance the trustworthiness of the study. Credibility of the study was enhanced by extensive iterative peer debriefing (i.e. regularly discussion of the results with the research team and the trainer) (Guba, 1981). Moreover, the individual interviews were done by an experienced interviewer who, in the course of the interviews, regularly clarified meanings of answers (Kvale, 1996). Furthermore, by providing comprehensive information about the context, participants and data collection methods of this study, transferability was increased (Kitto et al., 2008). The involvement of the researchers' perspective was accounted for by an analysis diary (conformability) (Mays and Pope, 2002).

\subsection{Ethical considerations}

The study was approved by the Medical Ethics Committee of the Atrium Medical Center, Heerlen, the Netherlands. All participants signed an informed consent form.

\section{Results}

\subsection{Characteristics of participants}

The 15 practice nurses who participated were all female and aged between 28 and 55 years. Their work experience ranged from 1.5 to 15 years and they all worked with people suffering from diabetes, COPD, asthma or cardiovascular diseases. Seven of the nurses also worked in elderly care and ten had already been exposed to some kind of training about self-management support (such as a training course on motivational interviewing). Ten patients, recruited from ten different nurses, were interviewed ( 7 women, 3 men). They were aged between 45 and 88 years, and their level of education ranged from elementary school to college of higher education. They suffered from diabetes, COPD or cardiovascular diseases. Thirteen patients ( 6 women, 7 men), aged between 41 and 75 years, participated in the audio-recordings of consultations. Nine of those patients suffered from diabetes, four patients suffered from cardiovascular diseases.

\subsection{Response}

Response rates differed between the data collection methods. All nurses participated in the individual interviews and filled in the questionnaire three times. The nine nurses who were invited to participate in the focus group all agreed to do so. Thirteen audio tapes of consultations with 13 different patients were delivered to the research team; four nurses delivered two audio tapes, five delivered one audio tape, six did not deliver any tapes. Ten nurses recruited a patient for an interview. Lack of time to inform patients, difficulties finding patients willing to participate and private circumstances were mentioned as reasons for not delivering an audio-tape or finding a patient for participation.

\subsection{To what extent was the approach implemented?}

Observationform (audiotapes)

We observed that none of the nine nurses who delivered an audiotape had fulfilled all performance objectives (see Table 1). Yet, all nurses fulfilled at least one of the behavioral determinants. Three nurses (out of nine) started their consultation by inviting the patient to ask questions and/or raise points for discussion. Five nurses asked about or discussed the patient's experience with regard to non-medical factors (two nurses discussed the patient's experience with regard to everyday activities/work, two nurses discussed the support patients had experienced from their environment and one nurse discussed the patient's experience with regard to everyday activities/work and the way he was coping with the condition). Two of these five nurses used the 4circles tool. We observed explicit goal formulation (during or at the end of a consultation) in three consultations (by three different nurses).

Questionnaire for practice nurses

Tables 2 and 3 show the results of the questionnaires on the practice nurses' current working method. Before the training course (t0), the nurses gave themselves relatively high scores for their current working method with regard to goal setting and action planning. The mean scores on all items increased slightly during the training period

Table 2

Questionnaire about current working method with regard to shared decision making about goals.

\begin{tabular}{|c|c|c|c|c|c|c|}
\hline \multirow[t]{2}{*}{ Items goal setting } & \multicolumn{2}{|l|}{ T0 } & \multicolumn{2}{|l|}{$\mathrm{T} 1$} & \multicolumn{2}{|l|}{$\mathrm{T} 2$} \\
\hline & Mean & Median & Mean & Median & Mean & Median \\
\hline 1. I explore patients' need for information. & 3.2 & 3 & 4 & 4 & 4 & 4 \\
\hline 2. I inform patients about topics that are relevant from my point of view. & 3.8 & 4 & 4 & 4 & 3.9 & 4 \\
\hline 3. I control if patients understand the information I gave. & 3.3 & 3 & 3.9 & 4 & 3.9 & 4 \\
\hline 4. I explore if patients experience any non-medical consequences of the condition (e.g. everyday life, work, social contacts). & 2.9 & 3 & 3.5 & 4 & 3.5 & 4 \\
\hline 5. I explore if patients accept their condition. (patient acceptance) & 2.3 & 2 & 2.8 & 3 & 3.1 & 3 \\
\hline 6. I explore if patients perceive their condition under control. (perceived control) & 3.6 & 4 & 3.7 & 4 & 3.9 & 4 \\
\hline 7. I explore patients' motivation to change. & 3.3 & 3 & 3.8 & 4 & 4.1 & 4 \\
\hline 8. I use patients' language when formulating goals. & 2.7 & 2 & 3.3 & 3 & 3.4 & 3 \\
\hline 9. I control if the patients' goals are clear. & 3.1 & 3 & 3.7 & 4 & 3.7 & 4 \\
\hline 10. I write goals down (in the registration system). & 3.2 & 3 & 3.6 & 3 & 4 & 4 \\
\hline 11. I differentiate between goals and actions. & 2.8 & 3 & 3.3 & 3 & 3.5 & 4 \\
\hline
\end{tabular}

$\mathrm{T} 0=$ Before the training, $\mathrm{T} 1=$ During the training, $\mathrm{T} 2=$ After the training.

( 1 = Never; 2 = Sometimes; 3 = Regularly; 4 = Often; 5 = Always $)$. 
Table 3

Questionnaire about current working method with regard to shared decision making about actions.

\begin{tabular}{|c|c|c|c|c|c|c|}
\hline \multirow[t]{2}{*}{ Items goal setting } & \multicolumn{2}{|l|}{ To } & \multicolumn{2}{|l|}{$\mathrm{T} 1$} & \multicolumn{2}{|l|}{$\mathrm{T} 2$} \\
\hline & Mean & Median & Mean & Median & Mean & Median \\
\hline 1. I made clear to my patient that a decision needs to be made. & 3.3 & 3 & 3.3 & 3 & 3.5 & 4 \\
\hline 2. I wanted to know exactly from my patient how he/she wants to be involved in making the decision. & 3.4 & 3 & 3.7 & 4 & 4.0 & 4 \\
\hline 3. I told my patient that there are different options for treating his/her medical condition. & 3.9 & 4 & 4.1 & 4 & 4.2 & 4 \\
\hline 4. I precisely explained the advantages and disadvantages of the treatment options to my patient. & 3.5 & 4 & 3.5 & 4 & 4.1 & 4 \\
\hline 5. I helped my patient understand all the information. & 3.7 & 4 & 3.9 & 4 & 4.1 & 4 \\
\hline 6. I asked my patient which treatment option he/she prefers. & 3.9 & 4 & 4.2 & 4 & 4.1 & 4 \\
\hline 7. My patient and I thoroughly weighed the different treatment options. & 3.3 & 3 & 3.5 & 3 & 3.8 & 4 \\
\hline 8. My patient and I selected a treatment option together. & 3.6 & 4 & 3.9 & 4 & 4.0 & 4 \\
\hline 9. My patient and I reached an agreement on how to proceed. & 4.1 & 4 & 4.2 & 4 & 4.1 & 4 \\
\hline 10. I write agreements down (in the registration system). & 4.1 & 4 & 4.4 & 4 & 4.3 & 4 \\
\hline
\end{tabular}

$\mathrm{T} 0=$ Before the training, $\mathrm{T} 1=$ During the training, $\mathrm{T} 2=$ After the training.

$(0=$ Completely disagree, $1=$ Strongly disagree, $2=$ Somewhat disagree, $3=$ Somewhat agree, $4=$ Strongly agree, $5=$ Completely agree) .

(comparing scores before and after the training course and during and after the training course). The greatest increase (difference in mean scores before and after training course equal to or higher than 0.8 ) was found for the exploration of patients' need for information (item 1), exploring if patients accepted the disease (item 5), exploring patients' motivation to change (item 7) and writing down goals (item 10). The item about exploring the patient's experiences with any non-medical consequences of the condition (item 4) had the lowest scores before the training course. After the training course, the score was still given the second lowest score.

\subsection{How did professionals experience the approach and the training course?}

Interviews and focus group

\subsubsection{More tailored coaching}

All practice nurses reported having experienced the profiles as highly recognizable in everyday practice. They reported that the profiles model had supported them in looking beyond the patient's medical condition, in paying more attention to the 'person behind the patient' and in setting more personalized goals. It was especially when confronted with patients who showed resistance to changing lifestyle behaviors that the profiles model was found to be very useful. In these cases, the nurses used the model to assess the reasons for resistance (e.g. does the patient accept the disease?) and adjusted their approach accordingly (e.g. by setting less demanding goals).

If you have a difficult patient, somebody who resists any changes to his lifestyle, you can look at the profiles and then you know that you have to take care not to require too much of the patient, but to compliment them on small changes.

\subsubsection{More exploration of the patient's perspective}

All nurses felt that by using the approach they had paid more attention to exploring the patients' perspectives, and had thereby gathered more relevant information about the patient. The nurses who tried out the 4-circles tool (12 out of 15 nurses) felt the tool had stimulated them to look beyond medical aspects. They perceived the use of the 4circles tool as providing greater insight into the patient's situation, leading to more realistic person-centered goals. The 4-circles tool was also valuable when coaching patients who, even after years of counselling, continued to have 'unhealthy' lifestyle habits. The nurses reported having used the tool to assess the factors keeping patients from changing their behavior (e.g. factors in the environment).

Look, people come here with their diagnosis of diabetes. Yet, that's not their whole story. With the four circles you can explore the bigger picture.
Three out of 15 nurses did not use the 4-circles model at all, as they did not feel the need to do so. They perceived they already knew their patients well enough and/or they perceived no need for a tool to explore the patient's perspective.

But it's not necessary for most of my patients. I've known them for a long time.

\subsubsection{Struggling to integrate the approach into routine care}

All nurses were struggling to integrate the approach into their usual work routines, where they mostly applied a protocol involving several medical measurements (like measuring blood pressure) and gave nontailored information about lifestyle requirements. Although the nurses were permitted (by the regional family medicine organization) to partly deviate from the protocol, they found it difficult to depart from it. They experienced the approach as a task additional to their usual working methods, and felt they had no time to explore the patient's perspective in depth. Some of the nurses reported that they only used the 4-circles tool when they knew they had time to allow their consultation to overrun by $10-15 \mathrm{~min}$.

Time is really an obstacle. I just did not find the time to have that kind of conversation with my patients. I also have to stick to my checklist.

\subsubsection{Difficult to use (in a flexible way)}

In addition to struggling to combine the approach with their usual work routines, the nurses also reported that the approach was difficult to use. With regard to the 4-circles tool, the nurses would have liked more practical guidance about its use (e.g. which questions can I ask?; for which patients can I use it?). Besides, they were afraid that using the 4-circles tool might raise topics that were not directly linked to the patient's condition, or questions about matters beyond their professional expertise. They experienced a lack of communication skills to handle those topics and questions.

The problem is that it is very open. You don't know what the patient is going to tell you. The conversation can go everywhere. I don't know if I can handle this.

Moreover, nurses found it very complex to use the patient profiles model when confronted with new patients. They were struggling to apply the model in a more flexible way.

I think it is difficult to adjust to the profiles in practice. On paper it's clear, but in practice it's much more difficult.

\subsubsection{Useful to have several exercise moments}

Practice nurses valued having several exercise moments during the training period, and being in constant contact with the coach who led 
the training course. Eight nurses emailed with or phoned the coach during the intervention period and experienced this as motivating.

It was nice to have that sort of incentive. Being in contact with her (the coach) via email encouraged me to keep going.

The other nurses did not use the opportunity to contact the coach. They reported that they had focused on their own strategies to learn the approach.

No, I did not use the opportunity to email her (the coach). I was too preoccupied with myself.

\subsubsection{Value of individual coaching}

The individual on-the-job coaching was most highly valued by the nurses. All but one of the nurses reported to have learned a lot about coaching patients in shared decision making during the on-the-job coaching. They especially highlighted the way the coach had provided (positive) feedback. They also mentioned that they felt safe, without any feeling of being judged. In addition, the coach's communication skills were highly appreciated. The nurses reported that they had learned most from observing how the coach communicated with and approached the patients.

She said that I was doing fine and that I have to realize that it's a learning process and that it is not possible to be perfect right away. That motivated me to keep going.

I watched her talk to a patient. She asked questions that I had never thought of. It was an eye-opener.

However, one nurse did not experience the coaching as valuable and safe. She mentioned that she felt being checked up on, and had been nervous and unable to handle the coach's feedback.

I really felt pressured. I think maybe I had the feeling that I have to prove that I can do it. And I did not know what to do with her feedback.

\subsection{How did professionals describe their own learning processes?}

Interviews

\subsubsection{Increased awareness of their own working methods, strengths, pitfalls} and beliefs

All nurses reported to have become more aware of and reflective about their current working method. They had gained insights into their strengths and pitfalls, as well as in the way their own beliefs/ attitudes influenced their work routines. This awareness had resulted in a greater ability to cope with stressful situations and with patients who show resistance to change.

It is very good to know what sort of people you're 'allergic' to. Throughout the whole process I learned that I particularly have trouble with know-it-all's. If you're aware of that, you can handle it better.

Twelve out of 15 nurses had realized that they frequently felt responsible for their patients' health situation and their progress in terms of behavior change. They had become more aware of the importance of being more reticent during consultations, giving the patients more chances to take responsibility for themselves.

And I also recognized that if you take a step back, you get more out of the patients themselves.

In addition, most of the nurses had been afraid that patients would find the approach too complex. They had worried that patients would be unable to answer their questions. They had also presumed that patients would not like the 4-circles model; they had been afraid that patients would find the colors and circles too childish. In addition, they had also thought that patients would sense their insecurity when applying the four circles. This had frequently prevented them from explicitly using the tool.

I thought maybe this is too vague for him. Maybe he doesn't know what to say.

\subsubsection{Increased awareness of process of behavior change}

Practice nurses had also become more aware of the process of behavior change. They reported that they had learned that changing behavior takes times and that it is very complex for patients to change their lifestyle behaviors. They had become increasingly aware of the complexity of changing several behaviors at once.

I was always like 'Hey, you have diabetes, you really have to pay attention to your diet.' Now I'm much more aware how difficult that actually is.

\subsubsection{Difficulties in adjusting current work routines}

All practice nurses mentioned that their learning process was mostly hampered by the fact that they experienced the approach as very different to their usual way of working. Although they were permitted to deviate to some extent from the protocol, they often adhered to it. Several reasons for this were mentioned. Some nurses reported that their performance within their workplace is mostly evaluated based on their fully applying the protocol.

But the protocols, they're are really...I was saying to my colleague just yesterday 'Maybe we'll get a lower score now'. Because we're assessed on the number of items that we tick in the protocol.

Other nurses seemed to experience an internal role conflict. Although they acknowledged the importance of exploring the patient perspective and setting person-centered goals, they sometimes regarded the medical examinations as more important.

Maybe that's silly, but yeah, it's also important to measure the blood pressure, to check the blood values, etc. Yeah, sometimes I think that's my first priority as a nurse.

Some nurses also reported that they did not feel skilled enough. They lacked self-efficacy about changing their work routines, and therefore often stuck to the old protocol, which felt safe to them.

I felt that I was hampered by my own insecurity. I didn't dare to really use it.

\subsubsection{Need for more support}

Although the family physicians involved had been informed about the approach and the training course, the nurses felt a lack of interest in the content of the training on the part of the physicians. They felt that the family physicians had delegated the health coaching entirely to them, and that they were often not willing to discuss the new approach. The nurses felt that more exchange of experiences with family physicians and other colleagues would have supported them in applying the approach.

You know, they're very busy, and I think that is why they're not really interested. They say, okay, that's your task. I'm sure you'll do well.

All nurses further acknowledged a need for follow-up to the training course. They called for regular refresher courses and for regular exchanges of information with other practice nurses. They also mentioned their need for more feedback, so they could continue to learn to work according to the approach. Nearly all of the nurses would appreciate more on-the-job coaching.

I really would like to have more feedback, for example about how to set goals together with a patient etc. 


\subsection{How did patients experience the approach?}

Interviews with patients

\subsubsection{Differences noticed}

Out of ten patients, five had noticed no difference with regard to the practice nurse's working methods. They reported that the nurse had done the usual medical measurements and asked about their lifestyle.

No, I did not really notice any difference. She checked everything and yeah, just like always.

\subsubsection{More questions}

Three out of five patients who did notice a difference had the impression that the nurse had asked more questions about their experiences. These patients felt the nurse had listened to them more than before, and had the feeling that she had more time for them.

She was more like 'How do you see it?' What do you think you can do yourself?'. This was new.

\subsubsection{Value of the 4-circles tool}

Two patients had filled in the 4-circles tool together with their practice nurses, and had taken the tool home. They regarded the tool as valuable and reported increased insight into their situation, resulting in more concrete goals. One patient expressed her surprise about the tool at first, wondering where the consultation would be going; she had found the consultation very different from previous ones, but very valuable.

\section{It was really an eye-opener. I looked at it and I thought: wow, it's true.}

The other patient at first found it difficult to apply the 4-circles tool. She did not know what was expected of her. After the nurse had explained the circles, she was able to discuss them with her nurse.

I thought it was difficult. At first you think, 'what does she mean by this?'. But after she'd explained it to me, it became clearer.

\subsubsection{Shared decision making}

Nine patients did not remember whether their practice nurse had asked them what they wanted to achieve. They reported that no goals or action plans had been explicitly agreed on. Five of these patients said that the nurse had told them what they needed to do in the coming months (e.g. go for a walk every day).

She always tells me that I have to walk more or use the bike more often, to lose weight.

Two patients reported that a concrete action plan to lose weight had been agreed upon.

Yeah, we agreed that I would lose 2 kilos in the next few months. We agreed that I'll be going for a walk with a friend of mine three times a week.

\section{Discussion}

The aim of this study was to investigate how an approach, focusing on supporting practice nurses to coach patients in shared decision making about goals and actions, works in practice. We did this by examining the extent to which the approach was implemented, the nurses' and patients' experiences with the approach, as well as the nurses' learning processes. Overall, nurses reported that the approach supported them in coaching patients in shared decision making. Interviews with nurses revealed that they had become more aware of their own attitudes, working methods, strengths and pitfalls. They felt that they had more in-depth discussions with patients and that they worked more tailored. The questionnaires about nurses' current working method showed that nurses assessed themselves as having improved with regard to making shared decisions with their patients. Furthermore, practice nurses experienced the training course (especially the on-thejob coaching) as supportive for their learning process. They perceived that they had become more aware of their learning needs. In addition, they expressed the importance of integrating the approach into the whole family medicine practice and their need for more exchange with/ support from family physicians. However, although nurses regarded themselves as having made progress in their learning process, they also felt that they still struggled to integrate the approach in routine care. They mostly experienced the approach as additional and different to their protocol-based work routines. Although permitted (by the regional family medicine organization) to apply the protocols in a more flexible way, they found it difficult to deviate from the protocols and therefore experienced difficulties to apply the approach in a flexible way. The audiotaped-consultations confirmed this struggle and indicated that the practice nurses found it difficult to explore patients' experiences from a holistic point of view and to formulate goals and action plans together with their patients. In addition, out of ten patients that were interviewed, half of the patients did not notice a difference in their practice nurse's working method before and after the training course. Those patients who did experience a difference felt that they were more listened to, that they got more insights in their situation and that more concrete goals were set. Our results raise further questions about the complexity for practice nurses to coach patients in shared decision making in primary care.

The nurses taking part in our study felt hampered by a lack of skills and a lack of self-efficacy to put the approach into practice. They experienced the approach as complex and especially struggled with applying it in a flexible way. The literature also shows that health coaching is thought to require different (communication) skills and is experienced as much more complex than applying a fixed protocol (Poskiparta et al., 2001; Stacey et al., 2008). Professionals experience health coaching and shared decision making as broad and abstract concepts and therefore struggle to implement them in practice (Elwyn et al., 2000; Howard and Ceci, 2013). It is experienced as complex, because it requires them to act flexibly and to constantly reflect on the way each individual patient can be supported (Zoffmann et al., 2008). Researchers agree that professionals should be trained in health coaching and shared decision making (Stiggelbout et al., 2012). Although shared decision making is one of the core components of health coaching, we did not find an approach that especially focused on coaching patients in shared decision making. More general training programs for shared decision making and health coaching frequently lack a detailed description about content and intensity, as well as rigorous evaluations (Wolever et al., 2013; Thiab Diouf et al., 2016). Based on a systematic review about health coaching programs Wolever et al. (2013) suggest, that it might be important to train professionals in behavioral change theory, motivational and empowerment strategies and communication techniques. We included these elements and also included training methods that had been found to be effective in changing professionals' complex behaviors (i.e. on-the-job coaching, focusing on self-reflection) (Poskiparta et al., 1999). However, professionals in our study still felt a lack of communication skills. It might be important to incorporate more training at the nurses' workplaces, as learning communication skills is a continuous process in interaction with the clinical environment (Spouse, 2001; Salmon and Young, 2011).

In addition we found that the practice nurses' attitudes influenced the implementation. The nurses were frequently doubtful about their patients' abilities and motivation to set goals and formulate action plans. Literature about shared decision making suggests that patients vary substantially in their preferences and abilities regarding participating in decision making (Levinson et al., 2005; McKinstry, 2000; Say et al., 2006; Murray et al., 2007). Although patients' preferences may 
be influenced by a range of variables, such as demographics and diagnosis, these associations are not absolute and professionals should not make assumptions about a patient's motivation and/or desire to participate (Levinson et al., 2005; McKinstry, 2000). Patients' hesitation to participate in setting goals and drawing up action plans might also result from them being used to practice nurses who adopt the role of medical expert (Van Dijk-de Vries, 2015). Therefore, it might be relevant to assess individual patients' preferences regarding decision making, and to better inform them about the practice nurse's role as coach (Levinson et al., 2005; McKinstry, 2000).

In addition, practice nurses in our study often felt responsible for the patient's health situation. Although the individual coaching gave them insight into this, they found it difficult to change it. Health professionals' attitudes towards health coaching and self-management support as a predictor of behavior change has been frequently discussed in the literature (Blakeman et al., 2006, MacDonald et al., 2008; Kennedy et al., 2014; Been-Dahmen et al., 2015). Kennedy et al. (2014) suggested that practice nurses often view self-management support as timeconsuming and futile (Kennedy et al., 2014). In addition, the study by Been-Dahmen et al. (2015) found that nurses frequently considered medical management to be their core task. Focusing on the traditional role of medical expert/educator makes it difficult to establish a collaborative partnership with the patient (Been-Dahmen et al., 2015; Thorne et al., 2000; Wilson et al., 2006). Furthermore, nurses' sense of responsibility for improving their patients' health, and their sometimes unrealistic expectations as regards patients' behavior change, were found to result in them continuing to control the patients' process, which impedes health coaching and shared decision making (Wilkinson et al., 2016). A greater focus on training nurses in the principles of behavior change might help them lower their expectations and facilitate their role as health coaches (MacDonald et al., 2008)

Our study also revealed the influence of contextual factors. In primary care, the nurses' work is mostly driven by external targets. Achieving targets in terms of biomedical indicators leads to financial rewards for the primary care practice (Kennedy et al., 2014; MacDonald et al., 2008). As a result, routine care is characterized by a protocolbased system, which conflicts with a holistic perspective on the patient (Kennedy et al., 2014; Willard-Grace et al., 2015). Although in our study the regional family medicine organization encouraged the participants to deviate to some extent from the protocol, the nurses still felt that their organization (mostly the supervising physician) expected them to fully adhere to the protocol. As there was limited exchange between the practice nurses and the family physicians about the approach, the nurses felt left alone in their attempts to change their working method. It became apparent that the practice nurses, the family physicians and the regional family medicine organization frequently did not hold the same views with regard to the practice nurse's role in the organization. The literature suggests that primary care nurses need to be supported by their supervisors to shift from a diseaseoriented protocol-based work routine to a more holistic and flexible approach (Oelke et al., 2014). When nurses experience trust and autonomy in their practice, they probably feel more able to apply protocols in a flexible and tailored way (Kennedy et al., 2014; Oelke et al., 2014). To fulfil their role as health coaches, practice nurses might need to be autonomous practitioners, though also part of a team that supports them in putting their role into practice (Kennedy et al., 2014; Stacey et al., 2008). Changing the role of the practice nurse in primary care might therefore require more attention to developing a shared view of the nurse's role within the family medicine practice.

Creating this shared vision might be facilitated by an organizational learning approach. Organizational learning aims to increase an organization's capacity to jointly design their work routines and define roles and responsibilities within the organization (Carroll and Edmondson, 2002; Clarke and Copeland, 2003). Thus, it focuses not only on educating and stimulating the individual professional, but also on the whole organization. Ideally, organizational learning leads to an organizational culture in which professionals support each other's professional development, share their expertise, identify their learning needs and plan how such needs can be met (Spouse, 2001). This perspective on learning fits in with commonly used constructivist and social learning theories, which claim that professionals develop their identity through active participation in a community (Brown and Duguid, 1991; Mann, 2011).

\subsection{Strengths and limitations}

This study was subject to some limitations. As practice nurses who had shown interest in shared decision making before were included, it might be that only the most intrinsically motivated practice nurses were included. Selection bias may also have played a role in the inclusion of patients. As the patients were selected by the practice nurses, these patients may not be representative, as there is a risk that the nurses only selected patients they considered to be satisfied. Another limitation was that the qualitative data was not coded independently by two researchers. Yet, we applied extensive iterative peer debriefing (with the research team and the trainer) and an analysis diary was kept. Moreover, most of the quantitative data collection instruments were self-developed by the research team, as no standardized instruments were available. Although the questionnaire for nurses was pilot-tested for its clarity and although it was based on the framework for shared decision making about goals and actions (face-validity), it was not tested for validity. However, for the second part of the questionnaire we used a validated questionnaire (SDM-Q-Doc).

Another limitation is the low response rate regarding the request to supply audiotapes of consultations. The nine nurses who delivered one or more audiotapes might not be representative of the entire group of practice nurses. The fact that many of the nurses were struggling to implement the approach might explain their reluctance to supply audiotapes.

This study had some strengths as well. First, we systematically developed the approach using principles of Intervention Mapping (Bartholomew et al., 2011), based on a thorough needs assessment and close collaboration with all important stakeholders (professionals, patients and primary care organizations). Based on this, we especially aimed to provide professionals with practical guidance and tools that would enable them to tailor their work routines. However, it might be possible that our approach was experienced as complex as it incorporated many different elements and tools (shared decision making about goals and actions, tool to explore the patients' perspective and a tool for tailoring). On the other hand, we were aware of the complexity of health coaching and shared decision making and therefore offered a comprehensive training course for nurses. With regard to the methodology, we broadened the scope of our study by means of triangulation of data collection, combining quantitative and qualitative data collection methods (Boeije, 2010).

\subsection{Conclusion}

Overall, our results show that changing the role of practice nurses from medical experts to health coaches, focusing on shared decision making about goals and actions, is very complex and requires paying attention to the nurses' skills and attitudes, as well as to contextual factors. Our approach and training course supported nurses in their learning processes. We mostly observed a change in attitudes and an increased awareness for their learning needs. Yet, most practice nurses struggled to integrate the approach in routine care and to apply it in a tailored way. Our results indicate that more time and training is needed for the nurses' role transition. Moreover, next to training the individual professional, it might be worthwhile to also focus on organizational learning, in order to increase a primary care organization's capacity to learn and change work routines and roles in a collaborative process. Our results might inform developers of health coaching and shared 
decision making approaches and indicate the importance of further research into the development and evaluation of health coaching approaches that focus on shared decision making.

\section{Funding}

This work was supported by Stichting Innovatie Alliantie (PRO-336) and Zuyd University of Applied Sciences, Heerlen, the Netherlands.

Stichting Innovation Alliantie provided financial support for the conduct of the research.

Zuyd University of Applied Sciences provided financial support for the conduct of the research and for the preparation of the article.

\section{Conflicts of interest}

None.

\section{Appendix A. Supplementary data}

Supplementary data associated with this article can be found, in the online version, at https://doi.org/10.1016/j.ijnurstu.2017.12.005.

\section{References}

Barr, V.J., Robinson, S., Marin-Link, B., Underhill, L., Dotts, A., Ravensdale, D., Salivaras, S., 2003. The expanded chronic care model: an integration of concepts and strategies from population health promotion and the chronic care model. Hosp. Q. 7, 73-82.

Bartholomew, L.K., Parcel, G.S., Kok, G., Gottlieb, N.H., Fernandez, M.E. 2011. Planning Health Promotion Programs: An Intervention Mapping Approach, third ed. JosseyBass, San Francisco.

Been-Dahmen, J.M.J., Dwarswaard, J.E., Hazes, J.M.V., van Staa, A., Ista, E., 2015. Nurses' views on patient self-management: a qualitative study. J. Adv. Nurs. 71, 2834-2845. http://dx.doi.org/10.1111/jan.12767.

Bennett, H.D., Coleman, E.A., Parry, C., Bodenheimer, T., Chen, E.H., 2010. Health coaching for patients with chronic illness. Fam. Pract. Manag. 17, 24-29.

Blakeman, T., Macdonald, W., Bower, P., Gately, C., Chew-Graham, C., 2006. A qualitative study of GPs' attitudes to self-management of chronic disease. Br. J. Gen. Pract. 56, 407-414.

Bloem, S., Stalpers, J., 2012. Subjective Experienced Health as a Driver of Health Care Behavior Nyenrode Research Paper. Academic Theory. Nyenrode Business University.

Bodenheimer, T., Handley, M.A., 2009. Goal-setting for behavior change in primary care: an exploration and status report. Patient Educ. Couns. 76, 174-180.

Boeije, H.R., 2010. Analysis in qualitative research, first ed. Sage Publications Ltd.

Brown, J.S., Duguid, P., 1991. Organizational learning and communities of practice: towards a unified view of working learning and innovation. Organ Sci, 2, 40-57.

Carroll, J.S., Edmondson, A.S., 2002. Leading organisational learning in health care. Qual. Saf. Health Care 11, 51-56.

Clarke, D.J., Copeland, L., 2003. Developing nursing practice through work-based learning. Nurse Educ. Pract. 3, 236-244.

Corbin, J., Strauss, J., 1988. Unending Work and Care: Managing Chronic Illness at Home, first ed. Jossey-Bass, San Francisco.

De Sutter, A., De Maeseneer, J., Boeckxstaens, P., 2013. Empowering patients to determine their own health goals. Eur. J. Gen. Pract. 19, 75-76.

Dubois, K., \& Van Rij, E., 2016. http://duboisvanrij.nl/coachende-zorgprofessional/ (assessed: 02.04.17)

Elwyn, G., Edwards, A., Kinnersley, P., Grol, R., 2000. Shared decision making and the concept of equipoise: the competences of involving patients in healthcare choices. $\mathrm{Br}$. J. Gen. Pract. 50, 892-897.

Elwyn, G., Frosch, D., Thomson, R., Joseph-Williams, N., Lloyd, A., Kinnersley, P., et al., 2012. Shared decision making: a model for clinical practice. J. Gen. Intern. Med. 27 1361-1367.

Fortin, M., Lapointe, L., Hudon, C., Vanasse, A., Ntetu, A.L., Maltais, D., 2004 Multimorbidity and quality of life in primary care: a systematic review. Health Qual. Life Outcomes 2, 51. http://dx.doi.org/10.1186/1477-7525-2-51.

Friesen-Storms, J.H., Bours, G.J., van der Weijden, T., Beurskens, A., 2015. Shared decision making in chronic care in the context of evidence based practice in nursing. Int. J. Nurs. Stud. 52, 393-402.

Ghorob, A., 2013. Health coaching: teaching patients to fish. Fam. Pract. Manag. 20, 40-42.

Guba, E.G., 1981. Criteria for assessing the trustworthiness of naturalistic inquiries. ECTJ 29, 75-91.

Heiligers, P.J.M., Noordman, J., Korevaar, J.C., Dorsman, S., Hinstman, L., Van Dulmen, A.M., De Bakker, D.H., 2012. Knowledge Base: Practice Nurses in the GP Practices, Ready for the Future? Netherlands Institute for health Services research (NIVEL), Utrecht (in Dutch).

Howard, L., Ceci, C., 2013. Problematizing health coaching for chronic illness self-management. Nurs. Inq. 20, 223-231.

Hsieh, H.F., Shannon, S.E., 2005. Three approaches to qualitative content analysis. Qual.
Health Res. 15, 1277-1288.

Kennedy, A., Rogers, A., Bowen, R., Lee, V., Blakeman, T., Gardner, C., Morris, R., Protheroe, J., Chew-Graham, C., 2014. Implementing, embedding and integrating self-management support tools for people with long-term conditions in primary care nursing: a qualitative study International Journal of Nursing Studies. Int. J. Nurs. Stud. 51, 1103-1113.

Kitto, S.C., Chesters, J., Grbich, C., 2008. Quality in qualitative research Criteria for authors and assessors in the submission and assessment of qualitative research articles for the Medical Journal of Australia. MJA 188, 243-246.

Kriston, L., Scholl, I., Holzel, L., Simon, D., Loh, A., Harter, M., 2010. The 9-item shared decision making questionnaire (SDM-Q-9): development and psychometric properties in a primary care sample. Patient Educ. Couns. 80, 94-99.

Kvale, S., 1996. Interviews: An Introduction to Qualitative Research Interviewing, First ed. Sage Publications, Thousand Oaks California.

Laurent, M., Reeves, D., Hermens, R., Braspenning, J., Grol, R., Sibbald, B., 2005. Substitution of doctors by nurses in primary care. Cochrane Database Syst. Rev. 18, CD001271.

Lenzen, S.A., Daniëls, R., van Bokhoven, M.A., van der Weijden, T., Beurskens, A., 2015. Setting goals in chronic care: shared decision making as self-management support by the family physician. Eur. J, Gen. Pract. 21, 138-144.

Lenzen, S.A., van Dongen, J.J.J., Daniëls, R., van Bokhoven, M.A., van der Weijden, T., Beurskens, A., 2016. What does it take to set goals for self-management in primary care? A qualitative study. Fam. Pract. 33, 698-703.

Lenzen, S.A., Daniëls, R., van Bokhoven, M.A., van der Weijden, T., Beurskens, A., 2017. Disentangling Self-management Goal Setting and Action Planning Interventions: A Scoping Review. (Unpublished results).

Levinson, W., Audiey, K., Kuby, A., Thisted, R.A., 2005. Not all patients want to participate in decision making a national study of public preferences. J. Gen. Intern. Med. 20, 531-535.

Lorig, K.R., Holman, H., 2003. Self-management education: history, definition, outcomes, and mechanisms. Ann. Behav. Med. 26, 1-7.

Lorig, K., 2006. Action planning: a call to action. J. Am. Board Fam. Med. 19, 324-325.

MacDonald, W., Rogers, A., Blakeman, T., Bower, P., 2008. Practice nurses and the facilitation of self-management in primary care. J. Adv. Nurs. 62, 191-199. http://dx. doi.org/10.1111/j. 1365-2648.2007.04585.x.

Mann, K., 2011. Theoretical perspectives in medical education: past experience and future possibilities. Med. Edu. 45, 60-68.

Mays, N., Pope, C., 2002. Qualitative research in health care: assessing quality in qualitative research. Br. Med. J. 320, 50-52.

McKinstry, B., 2000. Do patients wish to be involved in decision making in the consultation? A cross sectional survey with video vignettes. BMJ 321, 867-871.

Moore, G.F., Audrey, S., Barker, M., Bond, L., Bonell, C., Hardeman, W., Moore, L., O'Cathain, A., Tinati, T., Wight, D., Baird, J., 2015. Process evaluation of complex interventions: medical research council guidance. BMJ 350. http://dx.doi.org/10. 1136/bmj.h1258.

Mortelmans, D., 2007. Qualitative Analysis With Nvivo, First ed. Acco Uitgeverij, Leeuwen (in Dutch).

Murray, E., Pollack, L., White, M., Lo, B., 2007. Clinical decision-making: patients' preferences and experiences. Patient Educ. Couns. 65, 189-196.

Oelke, N.D., Besner, J., Carter, R., 2014. The evolving role of nurses in primary care medical settings. Int. J. Nurs. Pract. 20, 629-635.

Poskiparta, M., Liimatainen, L., Kettunen, T., 1999. Nurses' self-reflection via videotaping to improve communication skills in health counseling. Patient Educ. Couns. 36, 3-11.

Poskiparta, M., Liimatainen, L., Kettunen, T., Paivi, K., 2001. From nurse-centered health counseling to empowermental health counseling. Patient Educ. Couns, 45, 69-79.

Rodenburg-Vandenbussche, S., Pieterse, A.H., Kroonenberg, P.M., Scholl, I., van der Weijden, T., Luyten, G.P., et al., 2015. Dutch translation and psychometric testing of the 9-Item shared decision making questionnaire (SDM-Q-9) and shared decision making questionnaire-Physician version (SDM-Q-Doc) in primary and secondary care. PLoS One 10, e0132158. http://dx.doi.org/10.1371/journal.pone.0132158.

Salmon, P., Young, B., 2011. Creativity in clinical communication: from communication skills to skilled communication. Med. Educ. 45, 217-226.

Say, R., Murtagh, M., Thomson, R., 2006. Patients' preference for involvement in medical decision making: a narrative review. Patient Educ. Couns. 60, 102-114.

Spouse, J., 2001. Work-based learning in health care environments. Nurse Educ. Pract. 1 , $12-18$

Stacey, D., Murray, M.A., Légaré, F., Dunn, S., Menard, P., O'Connor, A., 2008. Coaching to support shared decision making: a framework, evidence, and implications for nursing practice, education, and policy. Worldviews Evid. Based Nurs. 5 (1), 25-35.

Stiggelbout, A.M., Van der Weijden, T., De Wit, M.P., Frosch, D., Légaré, F., Montori, V.M., Trevena, L., Elwyn, G., 2012. Shared decision making: really putting patients at the centre of healthcare. Br. Med. J. 344. http://dx.doi.org/10.1136/bmj.e256.

Thiab Diouf, N., Menear, M., Robitaille, H., Painchaud Guérard, G., Légaré, F., 2016. Training health professionals in shared decision making: update of an international environmental scan. Pat. Educ. Coun. 99, 1753-1758.

Thorne, S.E., Ternulf Nyhlin, K., Paterson, B.L., 2000. Attitudes toward patient expertise in chronic illness. Int. J. Nurs. Stud. 37, 303-311.

Van Dijk-de Vries, A., 2015. Towards Integrated Nurse-led Self-management Support in Routine Diabetes Care. PhD Dissertation. Maastricht University (p. 186). https: $/ /$ cris.maastrichtuniversity.nl/portal/files/573394/guid-48083a29-ac17-4f7c-8f1a2b6ae680715b-ASSET2.0.

World Health Organization, 2010. International Classification of Functioning, Disability and Health (ICF). (assessed 10.09.16). http://www.who.int/classifications/icf/en/.

Wagner, E.H., Bennett, S.M., Austin, B.T., Greene, S.M., Schaefer, J.K., VonKorff, M., 2005. Finding common ground: patient-centeredness and evidence-based chronic illness care. J. Altern. Complement. Med, 11, 7-15. 
Wilkinson, M., Whitehead, L., Crowe, M., 2016. Nurses perspectives on long-term condition self-management: a qualitative study. J. Clin. Nurs. 25, 240-246. http://dx. doi.org/10.1111/jocn.13072.

Willard-Grace, R., Chen, E.H., Hessler, D., DeVore, D., Prado, C., Bodenheimer, T., Thom, D.H., 2015. Health coaching by medical assistants to improve control of diabetes, hypertension, and hyperlipidemia in low-Income patients: a randomized controlled trial. Ann. Fam. Med. 13, 130-138. http://dx.doi.org/10.1370/afm.1768.

Wilson, P.M., Kendall, S., Brooks, F., 2006. Nurses' responses to expert patients: the rhetoric and reality of self-management in long-term conditions: a grounded theory study. Int. J. Nurs. Stud. 43, 803-818.

Wolever, R.Q., Simmons, L.A., Sforzo, G.A., Dill, D., Kaye, M., Bechard, E.M., Southard, M.E., Kennedy, M., Vosloo, J., Yang, N., 2013. A systematic review of the literature on health and wellness coaching: defining a key behavioral intervention in healthcare. Glob Adv Health Med. 2, 38-57.

Yach, D., Hawkes, C., Gould, C.L., Hofman, K.J., 2004. The global burden of chronic diseases: overcoming impediments to prevention and control. JAMA 291 (21), 2616-2622.

Zoffmann, V., Harder, I., Kirkevold, M., 2008. A person-Centered communication and reflection model: sharing decision-making in chronic care. Qual. Health Res. 18, 670-685.

van de Pol, M.H.J., Fluit, C.R.M.G., Lagro, J., Slaats, Y.H.P., Olde, R.M.G.M., LargoJanssen, A.L.M., 2016. Expert and patient consensus on a dynamic model for shared decision-making in frail older patients. Patient Educ. Couns. http://dx.doi.org/10. 1016/j.pec.2015.12.014. 